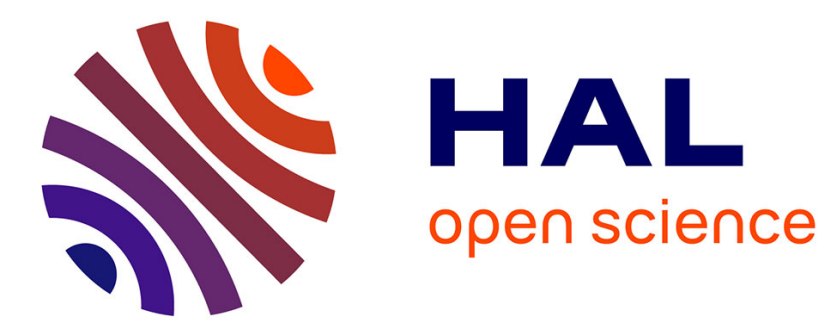

\title{
Universality of hydrogen bond distributions in liquid and supercritical water
}

\author{
Andrey G. Kalinichev
}

\section{To cite this version:}

Andrey G. Kalinichev. Universality of hydrogen bond distributions in liquid and supercritical water. Journal of Molecular Liquids, 2017, 241, pp.1038-1043. 10.1016/j.molliq.2017.06.114 . in2p301577627

\section{HAL Id: in2p3-01577627 \\ https://hal.in2p3.fr/in2p3-01577627}

Submitted on 9 Oct 2018

HAL is a multi-disciplinary open access archive for the deposit and dissemination of scientific research documents, whether they are published or not. The documents may come from teaching and research institutions in France or abroad, or from public or private research centers.
L'archive ouverte pluridisciplinaire HAL, est destinée au dépôt et à la diffusion de documents scientifiques de niveau recherche, publiés ou non, émanant des établissements d'enseignement et de recherche français ou étrangers, des laboratoires publics ou privés. 
Universality of hydrogen bond distributions in liquid and supercritical water

Andrey G. Kalinichev, ${ }^{1,2, *}$

${ }^{1}$ Laboratoire SUBATECH (UMR-6457), Institut Mines-Télécom Atlantique, 44307 Nantes, France

${ }^{2}$ International Laboratory for Supercomputer Atomistic Modelling and Multi-Scale Analysis, National Research University Higher School of Economics, Moscow, Russia

* Corresponding author: kalinich@subatech.in2p3.fr

Key words: $\quad$ supercritical water; computer simulations; hydrogen bonding; percolation; water structure; molecular dynamics 


\begin{abstract}
Monte Carlo and molecular dynamics computer simulations using the rigid TIP4P and the flexible $\mathrm{BJH}$ intermolecular $\mathrm{H}_{2} \mathrm{O}$ potentials were carried out for 50 states of supercritical water characterizing a very wide range of thermodynamic conditions, $573 \leq T \leq 1273 \mathrm{~K}$; $0.02 \leq \rho \leq 1.67 \mathrm{~g} / \mathrm{cm}^{3} ; 10 \leq P \leq 10000 \mathrm{MPa}$. Good agreement with available experimental data of the simulated thermodynamic and structural properties give confidence to the quantitative statistical analysis of intermolecular hydrogen bonding under the conditions studied. Energetic, geometric, and angular characteristics of supercritical H-bonds and their distributions at a given temperature remain almost invariant over the entire density range studied from dilute gas-like $\left(\sim 0.03 \mathrm{~g} \cdot \mathrm{cm}^{-3}\right)$ to highly compressed liquid-like $\left(\sim 1.5 \mathrm{~g} \cdot \mathrm{cm}^{-3}\right)$ states. The increase of temperature from ambient to supercritical affects the characteristics of $\mathrm{H}$-bonding in water much more dramatically than the changes in density along any supercritical isotherm. Compared to H-bonds in liquid water under ambient conditions, the H-bonds at $773 \mathrm{~K}$ are, on average, 10\% weaker, 5\% longer, and less linear.
\end{abstract}

Both above and below the H-bonding percolation threshold the fractions of $\mathrm{H}_{2} \mathrm{O}$ molecules involved in a certain number of H-bonds in liquid and supercritical water closely follow the universal binomial distribution as a function of the average number of $\mathrm{H}$-bonds per one water molecule in the system, as predicted by the independent bond theory. This universal distribution remains intact even when dynamic criteria of $\mathrm{H}$-bonding lifetimes are additionally applied. 


\section{Introduction}

The thermodynamic and transport properties of any substance near and above its critical point differ from those of the corresponding gas or liquid. Supercritical water (SCW) and supercritical aqueous solutions are especially important in this respect. Due to the extremely high compressibility, thermal expansivity, and heat capacity of SCW, small changes in pressure and/or temperature generate very substantial changes in density and enthalpy. These, in turn, affect diffusivity, viscosity, thermal conductivity, dielectric, and solvation properties of water, thus dramatically influencing the kinetics and mechanisms of chemical reactions [1].

In nature, hot aqueous solutions (known in the geological literature as hydrothermal fluids) penetrate the porous structure of the Earth's crust and play a key role in the transport and enrichment of ore-forming minerals [2]. Geological processes of hydrothermal fluids encompass a wide range of depths (i.e., pressures and temperatures), leading to complex physical and chemical behavior, such as the chemistry and hydrodynamics of so called "black smokers" (hot hydrothermal vents at the sea floor). SCW is even implicated in some thermodynamic scenarios leading to the origin of life on our planet [3].

From an engineering standpoint, SCW is also attracting rapidly growing attention as a promising medium for a wide range of environmentally friendly and energy-efficient technological applications from green chemical synthesis of new compounds and nanoparticles, to the production of synthetic fuels from biomass by catalytic conversion in SCW, and to the environmentally friendly disposal of toxic wastes by the process of supercritical water oxidation [1,4]. SCW can serve as a tunable solvent, its properties becoming closer to those of commonly used organic solvents, because of the flexibility of its density and dielectric constant. The same substance, water, may thus serve as both an "aqueous" and a "non-aqueous" solvent, having the advantage that all the reaction and separation processes occur in the same medium, tuned to desired characteristics by simply controlling its temperature and/or pressure [4]. 
It is now well established that many unique and anomalous properties of water, including its unusually high critical temperature, are the results of relatively strong specific hydrogen bonding between the $\mathrm{H}_{2} \mathrm{O}$ molecules [5]. In normal liquid water, such anomalous behavior as, for instance, the density maximum at $4^{\circ} \mathrm{C}$, arise from the competition between nearly ice-like tetrahedrally coordinated local H-bonding patterns and more compact molecular arrangements characterized by a higher proportion of weak and broken H-bonds (e.g., [5,6]). Under supercritical conditions, the molecular picture linking the structure and properties of water becomes more complicated. Nevertheless, a significant clarification of this picture has gradually emerged over the last 15-20 years due to the concerted efforts of many experimental [7-17] and computational molecular modeling [1827] research groups.

The question of the ranges of temperature and density (or pressure) where H-bonding interactions can significantly affect the observable properties of water has long been considered very important for the construction of realistic structural models for this fluid, and the first X-ray diffraction experiments on dense supercritical water by Yu.E.Gorbaty already gave significant quantitative insight [7]. This conclusion was later confirmed by the early molecular computer simulations of supercritical water [18-20] as well as by several other experimental techniques [8-10, 13-15]. However, it is important to remember that none of the experimental methods provides a direct way to measure the degree of H-bonding in SCW. There is always some level of uncertainty associated with the definition of what constitutes a hydrogen bond between two water molecules, as observed experimentally, hence with any quantification of the extent of H-bonding in SCW from experimental data. Numerical estimates of the number of $\mathrm{H}$-bonds per water molecule, $n_{\mathrm{HB}}$, are necessarily based on some assumptions and interpretation of experimental data in terms of particular molecular models.

Therefore, computational molecular modeling efforts are playing an especially important role in developing a detailed quantitative picture of hydrogen bonding in SCW as a locally heterogeneous system where the percolating network of H-bonds may be generally broken, but significant fractions 
of smaller patches (or clusters) of H-bonded molecules can still exist surrounded by a growing number of non-bonded $\mathrm{H}_{2} \mathrm{O}$ monomers with increasing temperature and decreasing density [28-34].

In this paper an attempt is made to re-analyze the results of Monte Carlo (MC) and molecular dynamics (MD) computer simulations in order to extract certain structural and topological parameters of such H-bonding networks and their local patches in SCW, which remain surprisingly universal over extremely wide ranges of temperature and density from a highly dilute supercritical water vapor to a highly compressed SCW at a density of up to $150 \%$ of normal bulk liquid water.

\section{Simulation Details}

The MC simulations were performed for a system of $N=216 \mathrm{H}_{2} \mathrm{O}$ molecules using the NPTensemble algorithm described in detail elsewhere [19,23]. Water molecules were interacting via the effective site-site pair potential TIP4P [35], which uses the experimentally known geometry of the rigid monomer, and has four interaction sites. A total of over 40 thermodynamic states were simulated for liquid and supercritical water along four isotherms (573, 673, 773, and $1273 \mathrm{~K})$ within the pressure range from 10 to $10000 \mathrm{MPa}$, thus sampling a very wide density range between 0.02 and $1.67 \mathrm{~g} / \mathrm{cm}^{3}$.

The MD simulations were performed using a conventional NVE-ensemble algorithm. The densities were chosen to correspond to the pressure range of $25<P<3000 \mathrm{MPa}$. The flexible $\mathrm{BJH}$ water model [36], employed in the simulations, has already been proven to provide quite accurate thermodynamic, structural, diffusional, and vibrational properties of SCW [21,22]. The total potential is represented by separate intermolecular and intramolecular contributions and an isolated $\mathrm{H}_{2} \mathrm{O}$ molecule has the same geometry as the TIP4P model.

All pressure-temperature points probed by the present MC and MD simulations are presented in Figure 1 on the phase diagram of pure water. The simulated thermodynamic properties have already been demonstrated to agree well with available equation of state data, if one correctly accounts for the difference in the critical parameters of the molecular $\mathrm{H}_{2} \mathrm{O}$ used in the simulations model and real 
water [22, 23, 26, 30]. For completeness, these data are provided here in graphical and tabulated form as Supplementary Information. The simulated structure of supercritical water is also in good agreement with x-ray and neutron diffraction data, as discussed earlier [22, 23, 26, 30, 38].

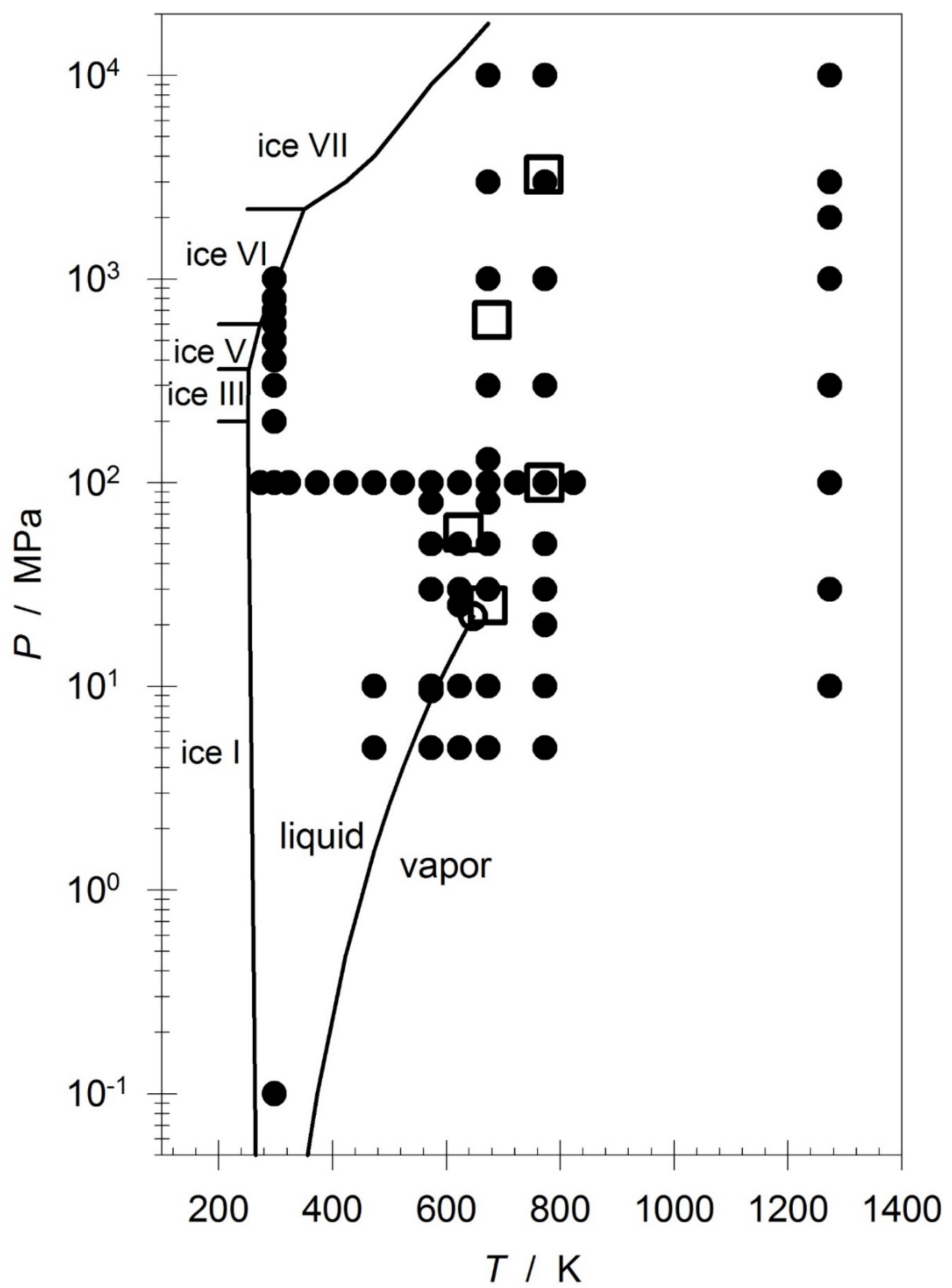

Figure 1. Phase diagram of water substance and thermodynamic $P$ - $T$-points simulated. Filled circles - MC simulations; open squares - MD simulations; open circle marks the critical point of water. 
This semi-quantitatively correct behavior of the simulated thermodynamic and structural properties of liquid and supercritical water over very wide ranges of temperatures, pressures, and denisties, gives a significant degree of confidence in developing a more detailed analysis of the local molecular geometric and energetic arrangements leading to H-bonding under supercritical conditions and their evolution with temperature and density.

\section{Hydrogen Bonding in Liquid and Supercritical Water}

To define an H-bond in molecular simulations is always simpler than in experimental measurements, because one always has a complete mechanical description of the modeled system in terms of the coordinates of all atoms and energies of interactions between them. Several criteria of H-bonding have been proposed, and a combination of a geometrical and an energetic one appears to be most useful, especially under supercritical conditions [39]. Kumar et al. have recently reviewed a number of H-bonding criteria available in the literature [40] and suggested the geometrical criteria, $R(\mathrm{O} \cdots \mathrm{H}) \leq 2.41 \AA$ and $\theta \geq 130^{\circ}$ for the $\mathrm{O}-\mathrm{H}^{\cdots} \cdots \mathrm{O}$ configuration, and an interaction energy between the hydrogen bonded water molecules to be more negative than $-12.9 \mathrm{~kJ} \mathrm{~mol}^{-1}$.

Here we are using a somewhat simpler combined geometric and energetic criterion of $\mathrm{H}$ bonding, based on the calculated radial distribution functions and intermolecular pair energy distribution [39], which can be formulated as follows: (1) the distance between the O-atom of the molecule-acceptor $i$ and the $\mathrm{H}$-atom of the molecule-donor $j$ is $R(\mathrm{O} \cdots \mathrm{H}) \leq 2.4 \AA$ (the maximum allowable H-bond length), and (2) the total energy of interaction between these two molecules $E_{i j}$ is below $E_{\mathrm{HB}}=-10 \mathrm{~kJ} / \mathrm{mol}$ (the upper limit of allowable H-bonding energy). This criterion of H-bonding is directly related to the two most natural parameters of any chemical bond — its length and strength.

Figure 2 presents the density and temperature dependencies of the average number of $\mathrm{H}$ bonds, $\left\langle n_{\mathrm{HB}}\right\rangle$, average H-bond energy $\left\langle E_{i j}\right\rangle_{\mathrm{HB}}$, distance $\left\langle R_{\mathrm{O}} \cdots \mathrm{H}\right\rangle_{\mathrm{HB}}$, and angle $\langle\theta\rangle=\langle\angle \mathrm{O}-\mathrm{H} \cdots \mathrm{O}\rangle$ of hydrogen bonds obtained from the MC simulations $[23,38]$ according to the above criterion. As it 
can be expected, the average number of $\mathrm{H}$-bonds per $\mathrm{H}_{2} \mathrm{O}$ molecule is systematically increases with increasing density along each isotherm, while the average length of H-bonds systematically decreases (Fig.2a,c). However, it is interesting to note that as the temperature increases the average total energy of H-bonding becomes smaller (less negative), but it is almost constant over the entire density range at any given temperature. The same can be said about the average HB angle $\langle\theta\rangle$ : despite some smooth variation with varying density, it also remains nearly constant over a very wide density range.
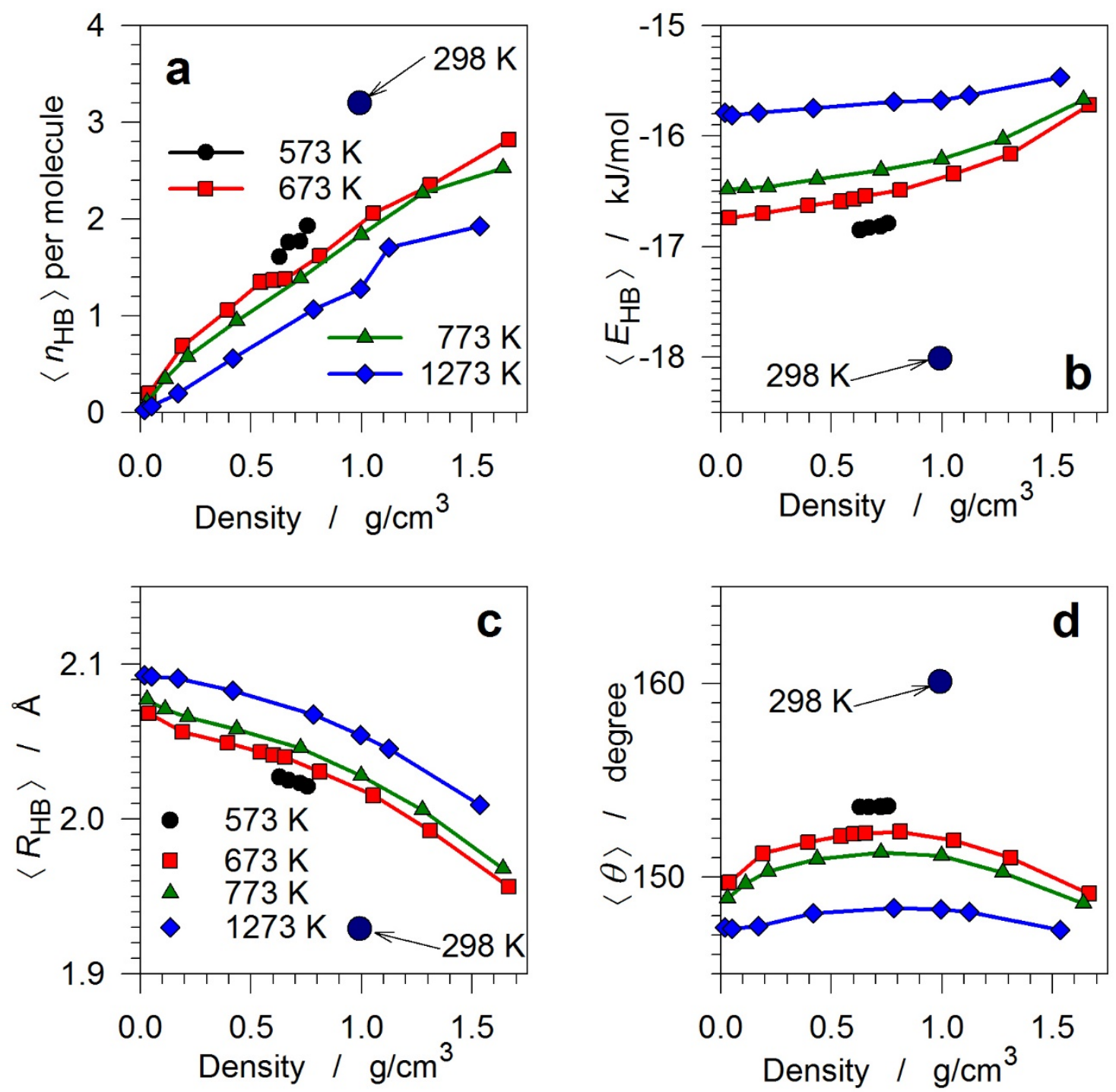

Figure 2. Average parameters of hydrogen bonds in liquid and supercritical water (after [26]). 
It is even more surprising that the distributions of H-boning angles $\theta$ are also virtually density independent along a supercritical isotherm. Figure 3 shows these distributions for the supercritical isotherm of $T=773 \mathrm{~K}$ together with the definition of the angles $\theta$ and $\varphi$ between two H-bonded molecules. These distributions are nearly identical for the states of supercritical water varying oders of magnitude in density, from dilute gas-like states $\left(\sim 0.03 \mathrm{~g} / \mathrm{cm}^{3}\right.$ at $\left.100 \mathrm{MPa}\right)$ to highly compressed liquid-like states $\left(\sim 1.27 \mathrm{~g} / \mathrm{cm}^{3}\right.$ at $\left.3000 \mathrm{MPa}\right)$.
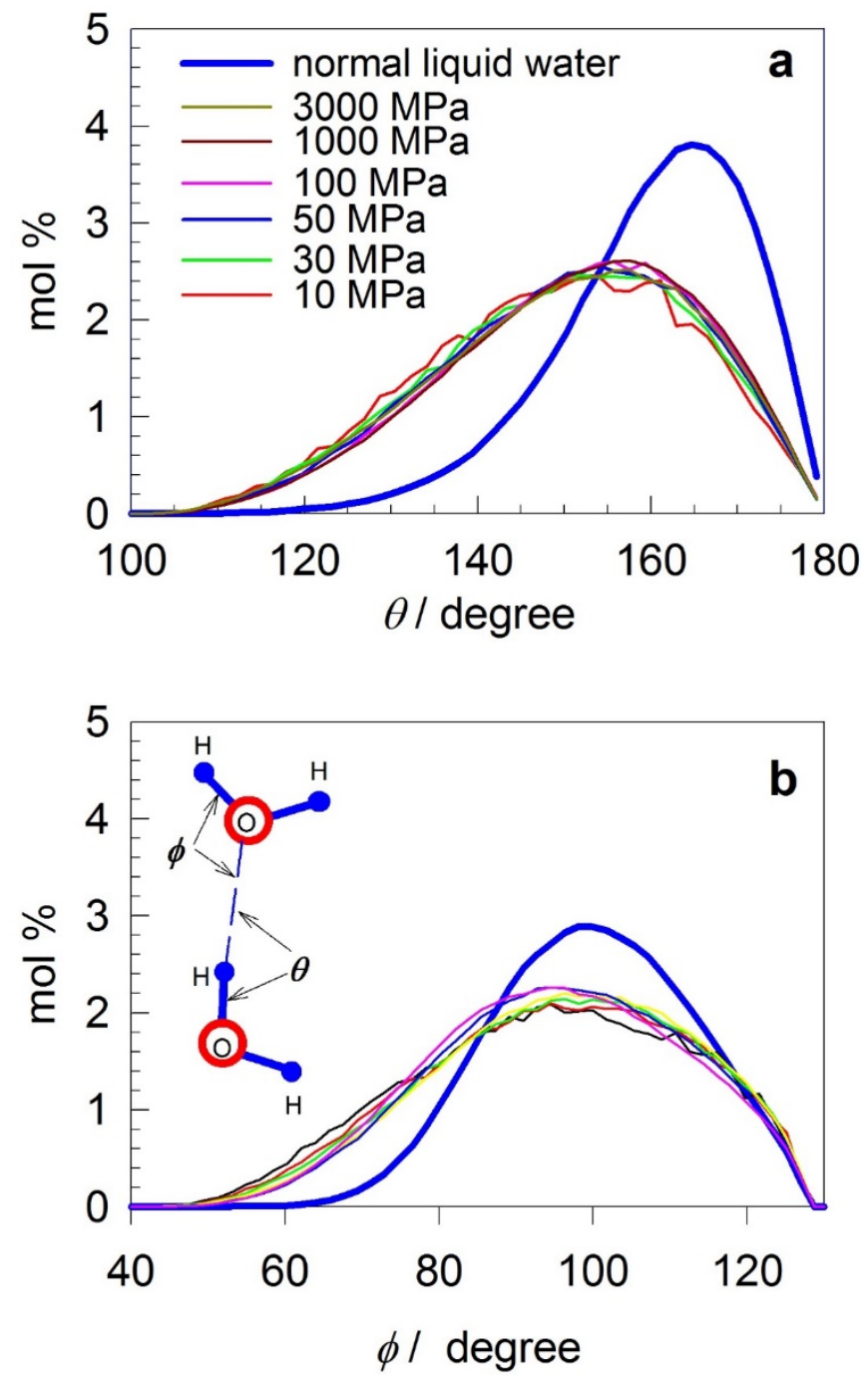

Figure 3. Distributions of $\mathrm{H}$-bonding angles $\theta$ and $\varphi$ in normal liquid water and along the supercritical isotherm of $T=773 \mathrm{~K}$.

This comparison makes it clear that under supercritical conditions the geometry of H-bonded molecular arrangements is much more strongly dependent on temperature than on density over a very 
broad range of thermodynamic states and remains nearly unique and universal for this particular temperature. One can consider this as an indirect indication that the increasing orientational disorder due to the rising temperature remains essentially the sole mechanism of H-bond breaking under supercritical conditions as it is already well established for normal liquid water [41, 42]. Another indirect indication of the reorientational mechanism of $\mathrm{H}$-bond breaking under supercritical conditions can be found in the librational spectra of $\mathrm{H}_{2} \mathrm{O}$ molecules calculated from MD simulations [21, 22, 31-33]. Even though these spectra are noticeably broadened under supercritical conditions, they still show the same characteristic librational frequencies in the range of $400-600 \mathrm{~cm}^{-1}$ as is observed in normal liquid water [43].

Most of the supercritical states at densities of $\rho \leq 1.0 \mathrm{~g} \cdot \mathrm{cm}^{-3}$ in Fig. 2a are characterized by $\left\langle n_{\mathrm{HB}}\right\rangle<1.6$, that is below the percolation threshold for the H-bonding network [44]. However, even though the continuous and infinite tetrahedral network of H-bonds is almost always broken in SCW, the fluid experiences significant local density inhomogeneities leading to the formation of H-bonded clusters of $\mathrm{H}_{2} \mathrm{O}$ molecules under these conditions [28-34].

It has long been established that the interconnected percolating network of H-bonds in liquid water can be quite accurately described by a simple binomial distribution

$$
f(i)=\left(\frac{m}{i}\right)\left(\frac{\left\langle n_{\mathrm{HB}}\right\rangle}{m}\right)^{i}\left(1-\frac{\left\langle n_{\mathrm{HB}}\right\rangle}{m}\right)^{m-i}
$$

where $f(i)$ is the fraction of molecules having $i \mathrm{H}$-bonds and $m$ is the maximum number of H-bonds an individual molecule can experience [44]. This distribution comes from a so-called independendbond theory and assumes that $m=4$ for an individual water molecule (two donated and two accepted H-bonds). It has been proven to hold also for moderately elevated temperatures and liquid-like densities [45] above the percolation threshold. However, the statistical analysis of our simulations shows that this theory is valid over much wider ranges of temperatures and densities covering the 
entire supercritical region, including the low density states well below the percolation threshold of $\left\langle n_{\mathrm{HB}}\right\rangle=1.6$.

Figure 4 shows the distribution functions $f(i)$ for all the supercritical thermodynamic states modeled by MC simulations as indicated on Fig.1. It is clear that the observed universality of H-bond distributions holds over the entire $P-\rho-T$ range studied. It is worth noting that similar universal relationships were recently observed by Idrissi et al. for liquid and supercritical methanol, where $m=3$ in Eq. (1).

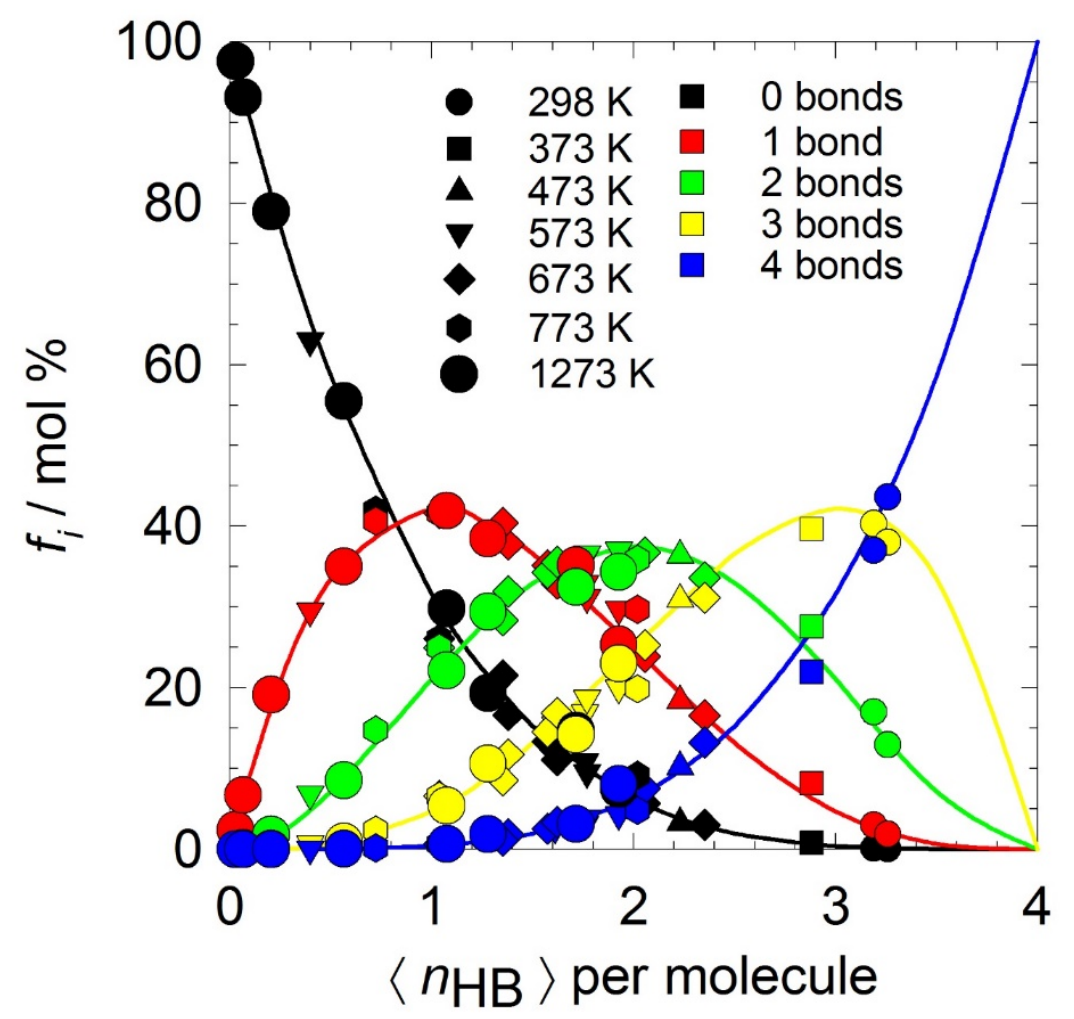

Figure 4. Distributions $f(i)$ of $\mathrm{H}_{2} \mathrm{O}$ molecules involved in $i \mathrm{H}$-bonds in liquid and supercritical water as a function of the average number of of H-bonds per molecule in the system, $\left\langle n_{\mathrm{HB}}\right\rangle$. Different symbols indicate different isotherms of MC simulations, while different colors indicate the $f(i)$ fractions with $i=0,1,2,3$, and 4 , respectively. The solid lines are calculated directly from Eq. (1).

The analysis of simulated H-bonding distributions discussed so far, was based on the instantaneous statistics of $\mathrm{H}$-bonds without any consideration of their lifetimes. However, MD simulations allow one to quantitatively analyze the effects of the H-bonding lifetimes on the observed 
distributions [47]. Several approaches have been developed [48-51]. Here we use the continuous definition of an H-bond [47] and introduce one additional parameter $\Delta \tau$ which is defined as follows. The MD-simulated trajectories can be recorded and analyzed with discrete time intervals (recording frequency). The continuous criterion assumes that an H-bond exists continuously if it satisfies all other geometric and energetic criteria of H-bonding at the beginning and the end of such interval, $\Delta \tau$. Obviously, the smallest value of $\Delta \tau$ is simply the time step of the particular MD simulation. However, manipulating $\Delta \tau$ within certain limits for the same MD trajectory one can make the dynamic criterion of $\mathrm{H}$-bonding more restrictive or less restrictive by allowing to ignore that the $\mathrm{H}$ bond can be actually broken and re-formed during this interval [49]. The estimates of H-bonding lifetimes in SCW of $\tau \approx 0.1-0.3$ ps obtained by this approach are comparable to other similar estimates $[32,33,50,51]$.

Figure 5 illustrates how the fractions $f(i)$ are distributed for the results of MD simulations shown in Fig.1. They, again, follow quite closely the predictions of the independent bond theory, Eq.(1). Even as more and more restrictive dynamic criterion of $\mathrm{H}$-bonding $\Delta \tau$ is applied, all the simulated distributions gradually shift to the left of Fig.5, but still continue to obey Eq.(1). The actual fractions $f(i)$ of $\mathrm{H}$-bonded molecules under the thermodynamic conditions closest to the critical point are presented in Table 1.

Table 1. Fractions $f(i)$ of H-bonded molecules in near-critical water at $T=630 \mathrm{~K}$ and $\rho=0.692 \mathrm{~g} / \mathrm{cm}^{3}$ depending on the dynamic criterion of H-bonding.

\begin{tabular}{|c|c|c|c|c|c|}
\hline$\Delta \tau / \mathrm{ps}$ & $\left\langle n_{\mathrm{HB}}\right\rangle$ & $f(0) / \%$ & $f(1) / \%$ & $f(2) / \%$ & $f(3) / \%$ \\
\hline 0.01 & 1.41 & 16.6 & 39.4 & 31.9 & 10.7 \\
\hline 0.1 & 0.83 & 40.2 & 40.2 & 16.3 & 3.0 \\
\hline 0.2 & 0.57 & 55.0 & 34.2 & 9.5 & 1.2 \\
\hline
\end{tabular}




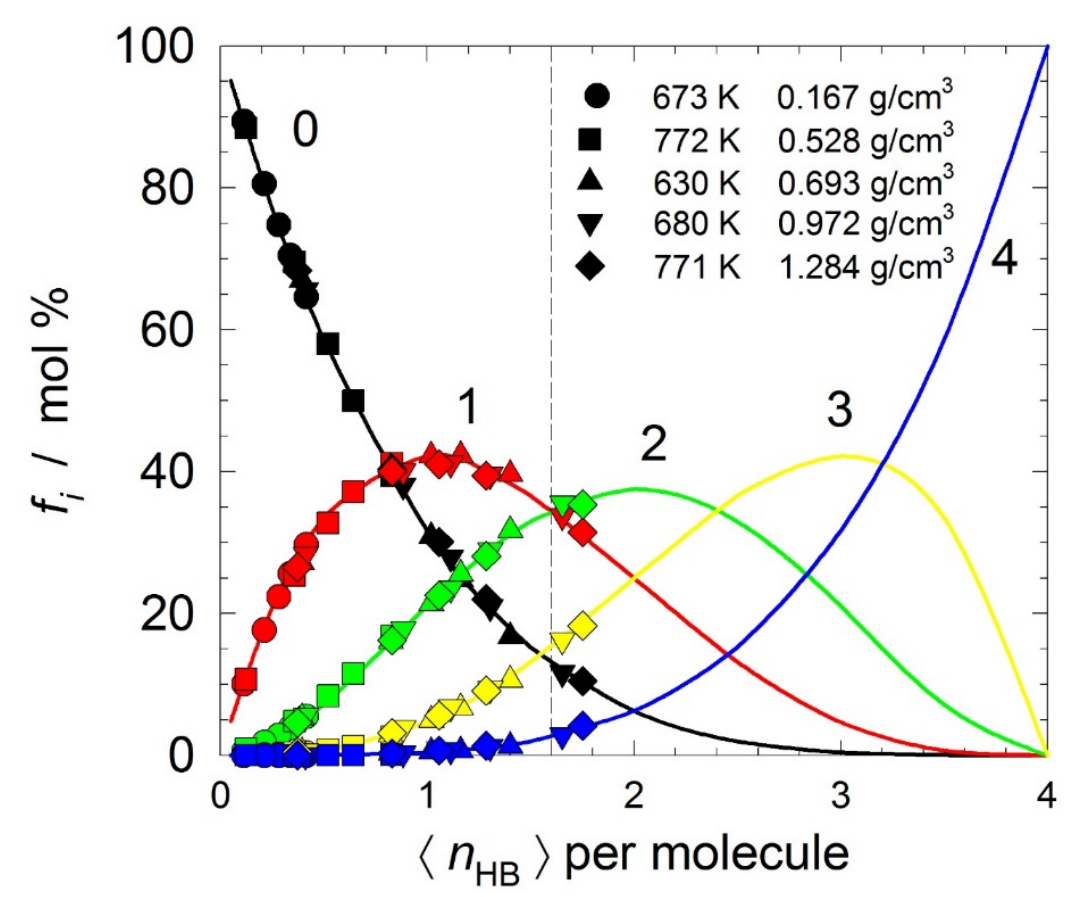

Figure 5. Distributions $f(i)$ of $\mathrm{H}_{2} \mathrm{O}$ molecules involved in $i \mathrm{H}$-bonds in liquid and supercritical water as a function of the average number of of H-bonds per molecule in the system, $\left\langle n_{\mathrm{HB}}\right\rangle$. Different symbols indicate different thermodynamic states of MD simulations, while different colors indicate the $f(i)$ fractions with $i=0,1,2,3$, and 4 , respectively. The solid lines are calculated directly from Eq. (1). The percolation threshold is shown as a thin vertical dashed line.

\section{Conclusions}

Application of the combined geometric and energetic H-bonding criterion [39] to the results of classical MC and MD simulations of SCW over very wide ranges of thermodynamic conditions shows that the average total energy of H-bonding in SCW is only weakly dependent on the density at any given temperature. The geometry of H-bonded molecular arrangements remains essentially the same over a very wide range of densities from dilute gas-like $\left(\sim 0.03 \mathrm{~g} \cdot \mathrm{cm}^{-3}\right)$ to highly compressed liquid-like $\left(\sim 1.5 \mathrm{~g} \cdot \mathrm{cm}^{-3}\right)$ fluid states at the same temperature. The increase of temperature from ambient to supercritical affects the characteristics of H-bonding in water much more dramatically than the changes in density along any supercritical isotherm. Compared to H-bonds in normal liquid water, $\mathrm{H}$-bonds at $773 \mathrm{~K}$ are, on average, almost $2 \mathrm{~kJ} \cdot \mathrm{mol}^{-1}$ weaker, have by $0.01 \mathrm{~nm}$ longer $\mathrm{O} \cdots \mathrm{H}$ bond distances, and are somewhat less linear. 
The distribution of fractions $f(i)$ of $\mathrm{H}_{2} \mathrm{O}$ molecules involved in $i \mathrm{H}$-bonds in liquid and supercritical water as a function of the average number of of $\mathrm{H}$-bonds per molecule in each particular thermodynamic state, $\left\langle n_{\mathrm{HB}}\right\rangle$ closely follows the universal binomial distribution as predicted by the independent bond theory. This universal distribution remains intact even when additional dynamic criteria of H-bonding lifetimes are applied.

\section{Acknowledgments}

This study has started many years ago when the author was still working at the Institute of Experimental Mineralogy of the Russian Academy of Sciences in Chernogolovka, near Moscow, and over the years it greatly benefited from many stimulating and thought inspiring discussions with Prof. Yu.E.Gorbaty. The initial financial support from the Russian Basic Research Foundation (grants 95-05-14748 and 97-03-32587), INTAS (grants UA-95-0096 and 96-1989), and CRDF (grant RC1170 to Y.E. Gorbaty), are most gratefully acknowledged, as well as the more recent support from the International Laboratory for Supercomputer Atomistic Modelling and Multi-Scale Analysis at the National Research University Higher School of Economics, Moscow, Russia, which finally allowed to bring the results of this research to publication.

\section{Supplemenrtary Information}

The calculated thermodynamic properties of liquid and supercritical TIP4P water from the present MC simulations in tabulated and graphical form are available the Internet from the journal's website. 


\section{References}

[1] Y.Marcus (2012) Supercritical Water. John Wiley \& Sons, Inc., 214p.

[2] H. L. Barnes (ed) (1997) Geochemistry of Hydrothermal Ore Deposits, 3rd ed., John Wiley \& Sons, New York, 1997, 972p.

[3] M.-P.Bassez (2003) Is high-pressure water the cradle of life? J. Phys.: Cond. Matter, 2003, L353-L361.

[4] A. Loppinet-Serani, C. Aymonier, F. Cansell (2008) Current and foreseeable applications of supercritical water for energy and the environment. ChemSusChem, 1, 486-503.

[5] J.L. Finney (2004) Water? What's so special about it? Philos. Trans .Royal Soc. B, 359, 11451165.

[6] G.G. Malenkov, D.L. Tytik, E.A. Zheligovskaya (2003) Structural and dynamic heterogeneity of computer simulated water: Ordinary, supercooled, stretched and compressed. J. Mol. Liquids, 106, 179-198.

[7] Y.E.Gorbaty, Y.N.Demianets (1983) The pair correlation functions of water at a pressure of 1000 bar in the temperature range 25-5000C. Chem. Phys. Lett., 100, 450-455.

[8] K. Yamanaka, T. Yamaguchi, H. Wakita (1994) Structure of water in the liquid and supercritical states by rapid x-ray diffractometry using an imaging plate detector. J. Chem. Phys., 101, 98309836.

[9] Y.E.Gorbaty, A.G.Kalinichev (1995) Hydrogen bonding in supercritical water. 1. Experimental results. J. Phys. Chem., 99, 5336-5340.

[10] A.K. Soper, F. Bruni, M.A. Ricci (1997) Site-site pair correlation functions of water from 25 to $400^{\circ} \mathrm{C}$ : Revised analysis of new and old diffraction data. J. Chem. Phys., 106, 247-254.

[11] Y.E.Gorbaty, R.B.Gupta (1998) The structural features of liquid and supercritical water. Ind. Eng. Chem. Res., 37, 3026-3035.

[12] Y.E.Gorbaty, G.V.Bondarenko, A.G.Kalinichev, A.V.Okhulkov (1999) The effect of pressure on hydrogen bonding in water: IR study of $v_{\mathrm{OD}} \mathrm{HDO}$ at pressures of up to $1500 \mathrm{bar}$. Mol. Phys., 96, 1659-1665.

[13] M. Nakahara, N. Matubayasi, C. Wakai, Y. Tsujino (2001) Structure and dynamics of water: from ambient to supercritical. J. Mol. Liquids, 90, 75-83.

[14] G.V. Bondarenko, Y.E. Gorbaty, A.V. Okhulkov, A.G. Kalinichev (2006) Structure and hydrogen bonding in liquid and supercritical aqueous $\mathrm{NaCl}$ solutions at a pressure of $1000 \mathrm{bar}$ and temperatures up to 500 degrees $\mathrm{C}$ : A comprehensive experimental and computational study. J. Phys. Chem. A, 110, 4042-4052.

[15] C.J.Sahle, C. Sternemann, C. Schmidt, S.Lehtola, S.Jahn, L.Simonelli, S.Huotari, M.Hakala, T. Pylkkänen, A.Nyrow, K.Mende, M.Tolan, K.Hämäläinen, M.Wilke (2013) Microscopic structure of water at elevated pressures and temperatures. Proc. Nat. Acad. Sci. USA, 110, 63016306.

[16] A.G.Novikov (2014) Topology and hydrogen connectivity in supercritical water. Physics of the Solid State, 56, 117-119. 
[17] Q. Sun, Q. Wang, D. Ding (2014) Hydrogen bonded networks in supercritical water. J. Phys. Chem. B, 118, 11253-11258.

[18] A.G.Kalinichev (1986) Monte Carlo study of the thermodynamics and structure of dense supercritical water. Intern. J. Thermophys., 7, 887-900.

[19] A.G.Kalinichev (1991) Monte Carlo simulations of water under supercritical conditions. I. Thermodynamic and structural properties. Z. Naturforsch., 46a, 433-444.

[20] P.T.Cummings, H.D.Cochran, J.M.Simonson, R.E.Mesmer, S.Karaborni (1991) Simulation of supercritical water and of supercritical aqueous solutions. J. Chem. Phys., 94, 5606-5621.

[21] A.G.Kalinichev (1993) Molecular dynamics and self-diffusion in supercritical water. Ber. Bunsenges. Phys. Chem., 97, 872-876.

[22] A.G.Kalinichev, K.Heinzinger (1995) Molecular dynamics of supercritical water: A computer simulation of vibrational spectra with the flexible BJH potential. Geochim. Cosmochim. Acta, 59, 641-650.

[23] A.G.Kalinichev, J.D.Bass (1997) Hydrogen bonding in supercritical water. 2. Computer simulations. J. Phys. Chem. A, 101, 9720-9727.

[24] A.A. Chialvo, E. Yezdimer, T. Driesner, P.T. Cummings, J.M. Simonson (2000) The structure of water from $25^{\circ} \mathrm{C}$ to $457^{\circ} \mathrm{C}$ : comparison between neutron scattering and molecular simulation. Chem. Phys., 258, 109-120.

[25] A.A.Chialvo, P.T.Cummings (1999) Molecular-based modeling of water and aqueous solutions at supercritical conditions. Adv. Chem. Phys., 109, 115-205.

[26] A.G. Kalinichev (2001) Molecular simulations of liquid and supercritical water: Thermodynamics, structure, and hydrogen bonding. Rev. Mineral. Geochem., 42, 83-129.

[27] J.M. Stubbs (2016) Molecular simulations of supercritical fluid systems. J. Supercrit. Fluids, 108, 104-122.

[28] R.D.Mountain (1999) Voids and clusters in expanded water. J. Chem. Phys., 110, 2109-2115.

[29] A.G.Kalinichev, S.V.Churakov (1999) Size and topology of molecular clusters in supercritical water: A molecular dynamics simulation. Chem. Phys. Lett., 302, 411-417.

[30] A.G. Kalinichev, S.V. Churakov (2001) Thermodynamics and structure of molecular clusters in supercritical water. Fluid Phase Equil., 183, 271-278.

[31] S. Krishtal, M. Kiselev, Y. Puhovski, T. Kerdcharoen, S. Hannongbua, K. Heinzinger (2001) Study of the hydrogen bond network in sub- and supercritical water by molecular dynamics simulations. Z. Naturforsch. A, 56, 579-584.

[32] E. Guàrdia, J. Martí (2004) Density and temperature effects on the orientational and dielectric properties of supercritical water. Phys. Rev. E, 69, 011502.

[33] I. Skarmoutsos, E. Guardia (2010) Effect of the local hydrogen bonding network on the reorientational and translational dynamics in supercritical water. J. Chem. Phys., 132, 074502074510 .

[34] L.B. Partay, P. Jedlovszky, I. Brovchenko, A. Oleinikova (2007) Percolation transition in supercritical water: A Monte Carlo simulation study. J. Phys. Chem. B, 111, 7603-7609. 
[35] W.L. Jorgensen, J. Chandrasekhar, J.D. Madura, R.W. Impey, M.L. Klein (1983) Comparison of simple potential functions for simulating liquid water. J. Chem. Phys., 79, 926-935.

[36] P. Bopp, G. Jancsó, K. Heinzinger (1983) An improved potential for non-rigid water molecules in the liquid phase. Chem. Phys. Lett., 98, 129-133.

[37] W. Wagner, A. Pruss (2002) The IAPWS formulation 1995 for the thermodynamic properties of ordinary water substance for general and scientific use. J. Phys. Chem. Ref. Data, 31, 387-535.

[38] A.G.Kalinichev, Y.E.Gorbaty, A.V.Okhulkov (1999) Structure and hydrogen bonding of liquid water at high hydrostatic pressures: Monte Carlo NPT-ensemble simulation up to 10 kbar. J. Mol. Liquids, 82, 57-72.

[39] A.G.Kalinichev, J.D.Bass (1994) Hydrogen bonding in supercritical water: a Monte Carlo simulation. Chem. Phys. Lett., 231, 301-307.

[40] R. Kumar, J.R. Schmidt, J.L. Skinner (2007) Hydrogen bonding definitions and dynamics in liquid water. J. Chem. Phys., 126, $204107-204112$.

[41] D. Laage, G. Stirnemann, F. Sterpone, J.T. Hynes (2012) Water jump reorientation: From theoretical prediction to experimental observation. Acc. Chem. Res., 45, 53-62.

[42] H. Ma, J. Ma (2011) Density dependence of hydrogen bonding and the translational-orientational structural order in supercritical water: A molecular dynamics study. J. Chem. Phys., 135, 054504.

[43] E. Spohr, G. Palinkas, K. Heinzinger, P. Bopp, M.M. Probst (1988) Molecular-dynamics study of an aqueous $\mathrm{SrCl}_{2}$ solution. J. Phys. Chem., 92, 6754-6761.

[44] R.L. Blumberg, H.E.Stanley, A.Geiger, P.Mausbach (1984) Connectivity of hydrogen bonds in liquid water. J. Chem. Phys, 80, 5230-5241.

[45] W. Nadler, T. Krausche (1991) Universality in hydrogen-bond networks. Phys. Rev. A, 44, R7888-R7890.

[46] A. Idrissi, R.D. Oparin, S.P. Krishtal, S.V. Krupin, E.A. Vorobiev, A.I. Frolov, L. Dubois, M.G. Kiselev (2013) The study of correlations between hydrogen bonding characteristics in liquid, sub- and supercritical methanol. Molecular dynamics simulations and Raman spectroscopy analysis. Farad. Disc., 167, 551-566.

[47] A. Luzar (2000) Resolving the hydrogen bond dynamics conundrum. J. Chem. Phys., 113, 10663-10675.

[48] T.I.Mizan, P.E.Savage, R.M.Ziff (1996) Temperature dependence of hydrogen bonding in supercritical water. J. Phys. Chem., 100, 403-408.

[49] A.G.Kalinichev, Y.E.Gorbaty (1998) The role of hydrogen bonding in the structure and properties of hydrothermal fluids. In: Zharikov V.A. (ed) Experimental and Theoretical Modeling of Mineral Formation Processes. Moscow, Nauka, pp. 242-264 (in Russian).

[50] B.S. Mallik, A. Chandra (2006) Hydrogen bond and residence dynamics of ion--water and water-water pairs in supercritical aqueous ionic solutions: Dependence on ion size and density. J. Chem. Phys., 125, 234502-234509.

[51] V. Petrenko, M. Antipova (2009) The mean lifetimes of H-bonds in water supercritical states. Russ. Journ. Phys. Chem. A, 83, 2243-2248. 